\title{
HIPODERMÓCLISE EM PACIENTES ONCOLÓGICOS SOB CUIDADOS PALIATIVOS
}

Eveline Treméa Justino' ${ }^{1}$ Fernanda Spiel Tuoto², Luciana Puchalski Kalinke, Maria de Fátima Mantovani ${ }^{4}$

\begin{abstract}
RESUMO: Trata-se de estudo descritivo, quantitativo e prospectivo com objetivo de descrever a experiência da utilização da hipodermóclise em pacientes sob cuidados paliativos e tratamento da dor, em hospital de referência em oncologia do Paraná. Foram acompanhados 16 participantes de novembro de 2008 a setembro de 2009, a coleta de dados foi realizada por meio de um instrumento estruturado e o acompanhamento aconteceu até a interrupção da utilização da hipodermóclise. A proporção de utilização da hipodermóclise entre os pacientes foi de $0,02 \%$, sendo mais comumente desenvolvida no ambulatório desta especialidade. A principal indicação foi o estágio avançado da doença $(56,25 \%)$ e o fármaco mais utilizado foi a morfina (93,75\%), a hipodermóclise foi usada em 31,25\% dos pacientes como via de hidratação. A utilização desta via alternativa para administração de fluidos em cuidados paliativos no hospital em questão é baixa, a adoção de protocolo padrão e divulgação podem contribuir para seu uso.
\end{abstract}

DESCRITORES: Enfermagem oncológica; Cuidados paliativos; Vias de administração de medicamentos; Saúde do adulto.

\section{HYPODERMOCLYSIS IN ONCOLOGY PATIENTS RECEIVING PALLIATIVE CARE}

ABSTRACT: This descriptive, quantitative and prospective study aimed to describe the experience of the use of hypodermoclysis in patients receiving palliative care and treatment for pain, in a hospital which is a center of excellence in oncology, in the state of Parana. 16 participants were monitored from November 2008 through to September 2009; data collection was undertaken using a structured instrument and monitoring took place up until the use of hypodermoclysis was interrupted. The proportion of use of hypodermoclysis among the patients was $0.02 \%$, and was carried out most commonly in the outpatient center of this speciality. The principal indication was the advanced stage of the disease (56.25\%) and the drug most used was morphine (93.75\%), and hypodermoclysis was used in $31.25 \%$ of the patients as a route of hydration. The use of this alternative route for administering fluids in palliative care in the hospital in question is low, and the adoption of a standard protocol and its dissemination could contribute to its use. DESCRIPTORS: Oncology nursing; Palliative care; Routes of administering medications; Adult health.

\section{HIPODERMÓCLISIS EN PACIENTES ONCOLÓGICOS BAJO CUIDADOS PALIATIVOS}

RESUMEN: Estudio descriptivo, cuantitativo y prospectivo cuya finalidad fue describir la experiencia de la utilización de la hipodermóclisis en pacientes en cuidados paliativos y tratamiento del dolor, en hospital de Paraná referencia en oncología. Fueron acompañados 16 participantes de noviembre de 2008 a septiembre de 2009; los datos fueron recogidos por medio de un instrumento estructurado y el acompañamiento ocurrió hasta la interrupción de la utilización de la hipodermóclisis. La proporción de utilización de la hipodermóclisis entre los pacientes fue de $0,02 \%$, siendo más comunmente desarrollada en el ambulatorio de esta especialidad. La principal indicación fue el estagio avanzado de la enfermedad $(56,25 \%)$ y el medicamento más utilizado fue la morfina (93,75\%), la hipodermóclisis fue usada en 31,25\% de los pacientes como fuente de hidratación. La utilización de esta fuente alternativa para administración de fluidos en cuidados paliativos en el hospital en cuestión es baja, la adopción de protocolo patrón y divulgación pueden contribuir para su uso.

DESCRIPTORES: Enfermería oncológica; Cuidados paliativos; Fuentes de administración de medicamentos; Salud del adulto.

${ }^{1}$ Enfermeira. Mestre em Enfermagem. Membro do Grupo de Estudos Multiprofissional em Saúde do Adulto da Universidade Federal do Paraná - GEMSA UFPR. Professora do Curso de Enfermagem da Faculdade União das Américas de Foz do Iguaçu - PR.

${ }^{2}$ Enfermeira Responsável pelo Setor de Cuidados Paliativos e Dor do Hospital Erasto Gaertner de Curitiba - PR. Especialista em Cuidados Paliativos e em Enfermagem Oncológica.

${ }^{3}$ Enfermeira. Doutora em Ciências da Saúde. Professora do Departamento e do Programa de Pós-Graduação em Enfermagem da UFPR. Membro do GEMSA.

${ }^{4}$ Enfermeira. Doutora em Enfermagem. Professora do Departamento e do Programa de Pós-Graduação em Enfermagem da UFPR. Membro do GEMSA.

Autor correspondente:

Luciana Puchalski Kalinke

Universidade Federal do Paraná

R. Pref. Lothário Meissner, 632 - 80210-170 - Curitiba-PR-Brasil

E-mail: lucianakalinke@yahoo.com.br
Recebido: 14/08/2012

Aprovado: 15/01/2013

Cogitare Enferm. 2013 Jan/Mar; 18(1):84-9 


\section{INTRODUÇÃO}

Novas alternativas tecnológicas na área da saúde e no desenvolvimento da terapêutica oncológica e dos Cuidados Paliativos têm sido adotadas, como técnicas e opções de administração de fluídos. A hipodermóclise é uma destas, porém, ainda é uma técnica pouco discutida e utilizada ${ }^{(1)}$ e consiste na administração de fluídos no espaço subcutâneo, de forma contínua ou intermitente. A utilização desta via para hidratação foi descrita, pela primeira vez, em 1914 em pacientes pediátricos $^{(2)}$. Mais de três décadas depois, em 1950, essa técnica caiu em desuso devido inadequada utilização, o que acarretou em resultados desastrosos ${ }^{(1)}$. Particularmente, em pacientes terminais, a utilização do método foi registrada em 1979, na Inglaterra, para o controle de êmese severa e obstrução intestinal ${ }^{(2)}$.

Trata-se de uma via alternativa utilizada, principalmente, em pacientes idosos e sob cuidados paliativos ${ }^{(3)}$, que frequentemente apresentam condições que impossibilitam a manutenção adequada de níveis de hidratação e nutrição, necessitando de vias alternativas para suporte clínico. A via intravenosa, geralmente, na fase avançada da doença encontra-se debilitada devido à terapêuticas medicamentosas, principalmente com agentes quimioterápicos, e às condições clínicas do paciente. Desta forma, a hipodermóclise pode ser considerada uma via de escolha para a reposição de fluidos, eletrólitos e alguns tipos de medicamentos, tanto no ambiente hospitalar quanto em atendimento domiciliar ${ }^{(4)}$.

A via subcutânea como alternativa para a administração de fluidos apresenta diversas vantagens, entre elas a comodidade para o paciente/família, simples punção e fácil administração, redução de custo e do tempo de internação, pouco desconfortável, baixa incidência de infecção, boa tolerância por pacientes agitados, diminuição dos efeitos sistêmicos adversos, redução da sobrecarga cardíaca e pode ser mantida por vários dias ${ }^{(3,5-8)}$. As desvantagens relacionadas ao uso da hipodermóclise são a instabilidade hemodinâmica, devido à infusão inadequada de grandes volumes em um período curto de tempo, e o uso de medicações e eletrólitos contra indicados para absorção no tecido subcutâneo ${ }^{(3,6-7,9)}$.

O foco deste trabalho está direcionado ao paciente oncológico sob cuidado paliativo, entendido como aquele voltado à qualidade de vida e alívio de sintomas de pacientes com doença maligna, progressiva e irreversível ${ }^{(3)}$. Dentro deste contexto, o objetivo desta pesquisa foi descrever aplicabilidade da hipodermóclise em cuidados paliativos em pacientes oncológicos em cuidados paliativos.

\section{MÉTODO}

Trata-se de uma pesquisa descritiva, prospectiva, com abordagem quantitativa desenvolvida em um hospital filantrópico, de ensino e referência na assistência a pacientes portadores de doenças oncológicas, localizado em Curitiba-Paraná.

A população do estudo correspondeu a todos os pacientes internados, em atendimento ambulatorial e/ou domiciliar e em acompanhamento pelo Serviço de Cuidados Paliativos e Dor (CPD), que utilizaram a hipodermóclise como alternativa de acesso a medicamentos e/ou soluções, no período compreendido entre 01 de novembro de 2008 a 30 de setembro de 2009.

Os critérios de inclusão foram: pacientes com idade superior a 18 anos, em acompanhamento pela equipe de CPD do hospital do estudo e com indicação médica e de enfermagem, de infusão subcutânea para hidratação e/ou administração de fármacos. Foram excluídos do estudo os pacientes que se encontravam com desidratação grave; risco de congestão pulmonar, insuficiência cardíaca congestiva e/ou distúrbio de coagulação, conforme avaliação médica.

Foram incluídos 16 pacientes, todos esses submetidos à técnica de hipodermóclise de 1 a 6 vezes, totalizando 30 procedimentos, o acompanhamento dos pacientes aconteceu durante todo período de utilização da hipodermólise. Para pacientes em atendimento domiciliar, foram realizadas visitas semanais para avaliação e troca da punção, para os pacientes em acompanhamento ambulatorial o retorno para avaliação foi agendado semanalmente. Quanto aos pacientes internados o acompanhamento foi diário, com orientação da equipe de enfermagem sobre o manuseio da técnica. A troca de punção foi realizada conforme a necessidade, e no máximo a cada 7 dias, de acordo com a rotina estabelecida na instituição.

Foi aplicado um instrumento de coleta de dados contendo 26 questões, que contemplavam informações de identificação, dados de internamento, atendimento ambulatorial ou domiciliar, e demais informações clínicas necessárias para a avaliação do procedimento, tais como: indicação da hipodermóclise, fluído e/ou medicamentos utilizados, local de infusão, troca de local e complicações. A coleta de dados foi realizada pela enfermeira do Serviço de CPD e os dados de identificação foram verificados no prontuário eletrônico e físico.

Após a obtenção dos dados, os pesquisadores se reuniram, com objetivo de realizar releitura das 
informações, discutindo e refletindo sobre cada item coletado. Os dados foram primeiramente organizados em planilhas utilizando o programa Excel $^{\circledR}$ e posteriormente analisados. As variáveis foram representadas por frequência absoluta (n) e relativa (\%).

Em relação aos aspectos éticos da pesquisa, o projeto foi aprovado pelo Comitê de Ética em Pesquisa (CEP) da instituição, sob o protocolo n. 1793, os procedimentos do estudo aconteceram somente após o aceite do participante, e assinatura do Termo de Consentimento Livre e Esclarecido, concordando com os termos da pesquisa.

\section{RESULTADOS}

Durante os 11 meses de coleta de dados, 1.410 atendimentos hospitalar, ambulatorial e domiciliar foram realizados pelo CPD e em 30(0,02\%) atendimentos foi aplicada a terapêutica subcutânea por meio da hipodermóclise (Tabela 1).

Entre os 16 participantes do estudo, 10 (62,5\%) eram mulheres e seis $(37,5 \%)$ eram homens, a idade mínima foi de 22 anos e a máxima de 95 , com média de 61 anos. Quanto ao diagnóstico oncológico, em três $(18,75 \%)$ a localização primária do tumor era

Tabela 1 - Taxa de utilização da hipodermóclise geral e por modalidade de atendimento. Curitiba, 2009

\begin{tabular}{lcccc}
\hline $\begin{array}{l}\text { Modalidade de } \\
\text { atendimento }\end{array}$ & $\begin{array}{c}\text { n. de instalação da } \\
\text { hipodermóclise }\end{array}$ & n. de atendimentos & $\begin{array}{c}\text { Taxa de utilização } \\
\text { por modalidade }\end{array}$ & $\begin{array}{c}\text { Taxa geral de } \\
\text { utilização }\end{array}$ \\
\hline Ambulatorial & 14 & 1088 & $0,01 \%$ & \\
Domiciliar & 11 & 152 & $0,07 \%$ & $0,02 \%$ \\
Hospitalar & 5 & 170 & $0,03 \%$ & - \\
Total & 30 & 1410 & - & - \\
\hline
\end{tabular}

no estômago; em dois $(12,50 \%)$ era, respctivamente cólon, pâncreas e próstata; em um $(6,25 \%)$ era, respectivamente, no colo uterino, no pulmão, de reto, um na vesícula biliar e um participante tinha diagnóstico de Sarcoma de Ewing. Dois pacientes não eram portadores de doença oncológica, estavam em acompanhamento pelo Serviço CPD para tratamento da dor, um deles apresentava fratura de cóccix e o outro condromalácia e bócio colóide.

Especificamente oito (50\%) pacientes foram submetidos ao procedimento mais de uma vez. Referente à modalidade de atendimento, 14 (46,66\%) aplicações da técnica foram executadas durante atendimento ambulatorial, $11(36,66 \%)$ no atendimento domiciliar e cinco $(16,66 \%)$ em atendimento hospitalar, os critérios de indicação estão apresentados na tabela 2.

Referente ao local escolhido para instalação da via e tendo 30 instalações de hiperdermóclise como referência, em $28(93,34 \%)$ a aplicação foi realizada em região infraclavicular, duas $(6,66 \%)$ na região abdominal, com variação entre o lado esquerdo (16; $53,33 \%)$ e direito $(14 ; 46,66 \%)$. Em todas as situações o dispositivo de escolha foi o cateter agulhado, com variações no calibre, o 21 Gauge $(\mathrm{G})$ foi utilizado em $12(40 \%)$ casos, $23 \mathrm{G}$ em seis (20\%), 25G em 11 $(36,66 \%)$, enquanto o calibre $27 \mathrm{G}$ foi utilizado em apenas um (3,33\%) caso.

O fármaco mais utilizado foi a morfina, prescrito para $15(93,75 \%)$ pacientes, administrada
Tabela 2 - Indicações para a instalação da hipodermóclise identificadas nos pacientes oncológicos sob cuidados paliativos. Curitiba, 2009

\begin{tabular}{lcc}
\hline Indicações & n & \% \\
\hline Náuseas e vômitos & 4 & 25 \\
Doença terminal & 4 & 25 \\
Náuseas e vômitos e doença terminal & 2 & 12,5 \\
Debilidade severa e doença terminal & 2 & 12,5 \\
Desidratação leve/moderada & 1 & 6,25 \\
$\begin{array}{l}\text { Náuseas e vômitos, dor severa e doença } \\
\text { terminal }\end{array}$ & 1 & 6,25 \\
$\begin{array}{l}\text { Dor de difícil controle com analgésico } \\
\text { por via oral }\end{array}$ & 1 & 6,25 \\
$\begin{array}{l}\text { Falta de medicação via oral na farmácia } \\
\text { do hospital }\end{array}$ & 1 & 6,25 \\
Total & 16 & 100 \\
\hline
\end{tabular}

isoladamente ou em combinação com outras medicações (Figura 1). A hipodermóclise como via para hidratação foi utilizada em cinco (31,25\%) dos 16 pacientes, sendo que em todas as situações a Solução Fisiológica (SF) a 0,9\% foi administrada; em três $(60 \%)$ aplicações em associação com Solução Glicosada a 5\% e em uma (20\%) aplicação houve a adição de duas ampolas de glicose a 50\%.

O período de uso da hipodermóclise variou de um a 55 dias, com média de 10,16 dias. Sete participantes permaneceram com a hipodermóclise por um período superior a sete dias, sendo necessária a mudança de 
local em intervalos regulares. Na ausência de sinais e sintomas de complicações, as trocas de local de punção foram realizadas com intervalo de sete dias. Em três pacientes houve a necessidade de mudança de local em período inferior, sendo que o motivo em duas situações foi uma discreta irritação no local do acesso e na terceira havia a presença de hematoma, o que corresponde a $10 \%$ das 30 vezes em que esta técnica foi empregada.

Dos 9 pacientes que em algum momento foram atendidos em domicílio, seis $(66,7 \%)$ receberam cui- dados de familiares para a manutenção do cateter, dois $(22,2 \%)$ além de receberem auxílio de familiares participam do cuidado com o cateter e apenas um $(11,1 \%)$ possuía um cuidador formal (auxiliar de enfermagem) para a realização dos cuidados necessários com a via.

Em relação ao motivo pelo qual foi interrompida a utilização da hipodermóclise, 13 (81,25\%) foram relativos ao óbito, um $(6,25 \%)$ não aceitou permanecer com a hipodermóclise e outro $(6,25 \%)$ concluiu o tratamento medicamentoso, momentaneamente, o que levou a retirada da hipodermóclise.

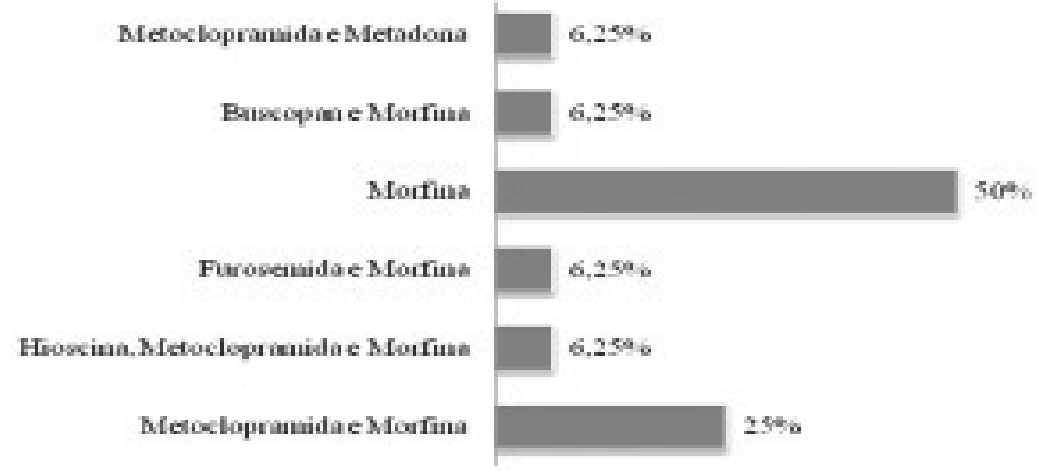

Figura 1 - Fármacos e combinações infundidas através da terapia subcutânea. Curitiba, 2009

\section{DISCUSSÃO}

As indicações para a escolha da via subcutânea para hidratação e/ou administração de fármacos são várias, porém sua utilização e publicações que incentivem o uso ainda são poucas. Foram encontrados alguns relatos da sua utilização, um deles na cidade de Singapura, no Dover Park Hospice, onde 19\% dos pacientes do serviço de cuidados paliativos utilizaram a via $^{(5)}$. Em outra pesquisa realizada no Centro de Saúde de Odivelas em Portugal, somente 3,5\% dos pacientes fizeram uso da via ${ }^{(10)}$. No entanto, a utilização da via na instituição estudada foi de apenas $0,02 \%$ de uso, o que reforça o quanto essa é pouco utilizada.

Possivelmente uma das razões de poucos estudos darem ênfase à utilização do hipodermóclise é de que a via oral ainda é a primeira opção para administração de medicamentos em pacientes em domicílio, ou sem condições de acesso venoso periférico. Porém, em casos de pacientes em fase terminal, a deglutição, muitas fezes está deficiente, o que torna difícil a utilização da via oral como alternativa de administração de medicamento e, principalmente, como via de hidratação.

Para os pacientes com câncer, náuseas e vômitos são sintomas significativos e podem ocasionar alterações psicofisiológicas, levando a diminuição da ingesta hídrica, alimentar e à intolerância à ingesta de medica mentos, contribuindo para o aumento da desidratação e a interrupção do tratamento. Estima-se que $50 \%$, ou mais, dos pacientes em tratamento quimioterápico, e um número similar destes com câncer avançado, experimentem estes sintomas ${ }^{(11)}$.

Nos casos de localização primária do tumor no sistema gastrointestinal, que era o caso de $9(56,25 \%)$ participantes do presente estudo, a indicação de hipodermóclise torna-se significativa devido à severidade das condições de acometimento dos órgãos, principalmente dificultando a alimentação e absorção das drogas. A evolução para a fase avançada do câncer, momento este extremamente importante para a indicação da assistência pelo Serviço CPD, para o alívio do sofrimento pela promoção do conforto em todas as esferas do cuidar (física, psíquica, social e espiritual), a hipodermóclise pode ser aplicada como parte deste atendimento.

Neste estudo, o estágio avançado da doença e os sintomas de náuseas e vômito, estiveram presentes em $9(56,25 \%)$ e $7(43,75 \%)$ pacientes, respectivamente; estas condições são benéficas para emprego da técnica. Os resultados obtidos remetem a outros estudos similares, como o realizado com 348 pacientes, também em cuidados paliativos, que apresentou como princi- 
pais indicações da hipodermóclise (50\%), a disfagia, náuseas e vômitos, agitação e prostração ${ }^{(10)}$. Em outro estudo envolvendo 83 pacientes com o diagnóstico de Síndrome da Imunodeficiência Adquirida as principais indicações para a hipodermóclise foram: fase avançada da doença, rede venosa ruim, disfagia, uso de opióides, náuseas e vômitos ${ }^{(1)}$.

Percebeu-se, em relação à escolha do local para instalação da hipodermóclise, que não houve a utilização de várias regiões que poderiam apresentar condições, talvez melhores, para a punção subcutânea. Principalmente por tratar-se de pacientes, na sua maioria, com déficit nutricional, o que acarreta em diminuição do tecido subcutâneo e maior intolerância as grandes infusões. Na realidade do serviço a região de escolha foi a infraclavicular e em apenas dois $(6,66 \%)$ a região abdominal. Esta última poderia ter sido mais utilizada por ser uma região com maior camada de tecido subcutâneo, o que proporcionaria benefícios ao paciente e cuidador, como maior visibilidade ao paciente, quando este realiza o autocuidado; além, de maior tolerância a grandes volumes.

$\mathrm{Na}$ literatura, os locais indicados para a punção subcutânea são: região infraclavicular; região infraescapular; região abdominal (flanco); face anterior, interna ou externa da coxa; região anterior e externa dos braços (deltóide) $^{(12-13)}$. Essas regiões admitem um volume bem acima do tolerado pela técnica tradicional de injeção subcutânea, que seria de até dois mililitros. Para a escolha do local da punção, deve-se levar em consideração, o volume de líquido a ser administrado. Ou seja, é importante que a região escolhida tenha quantidade de tecido subcutâneo para a infusão de maiores volumes, e a região abdominal é uma boa opção. Outras observações devem ser realizadas, como: evitar administração direta sobre o local do tumor, em membros com linfedema, em pele previamente irradiada, uma vez que o suprimento sanguíneo pode estar reduzido, em locais com infecção, fissuras ou hematomas e na região abdominal, quando presença de ascite ${ }^{(14)}$.

Em todas as situações o dispositivo de escolha foi o cateter agulhado. Ainda não está disponível dispositivo específico para o procedimento, porém, pode-se utilizar cateter agulhado ou não agulhado. Dentre os agulhados, o disponível é o escalpe, com variação no calibre de acordo com a quantidade de tecido subcutâneo do paciente e do volume a ser infundido; autores recomendam cateteres calibre 21 ao $27 \mathrm{G}^{(3,5,15)}$, sendo este último adequado para pacientes pediátricos ou caquéticos.
Seria preferível a utilização de cateter não agulhado, por ser flexível e apresentar menor risco de trauma cutâneo. Contudo, o custo elevado deste material tornaria o procedimento mais oneroso e, consequentemente, menos realizado. Estudo demonstrou que alguns pacientes referiram não perceber a diferença entre os dispositivos quando posicionados corretamente ${ }^{(15)}$.

$\mathrm{O}$ fármaco mais utilizado foi a morfina, prescrito para 15 (93,75\%) pacientes, administrada isolada ou em combinação com outras drogas. Resultado similar foi descrito em um estudo desenvolvido no Hospital Universitário de Genebra - Suíça ${ }^{(16)}$.

Nos cinco pacientes em que a hipodermóclise foi utilizada como via para hidratação, as soluções administradas foram fisiológico, glicosada e glicose hipertônica. As soluções melhor toleradas são as isotônicas e as hidrossolúveis, por serem menos irritantes e apresentar menor risco de acumulação; soluções muito ácidas ou básicas podem aumentar o risco de precipitação ou irritação $0^{(17)}$. Estudos demonstram que a solução glicosada a 5\% é isenta de riscos ${ }^{(13)}$.

Sobre o volume de fluídos, alguns autores relatam que este não deve exceder a $2.000 \mathrm{ml}$ em 24 horas $^{(3,6)}$. Entretanto, outros autores citam que é possível a administração de até $1.500 \mathrm{ml} /$ dia em cada sítio de punção, sendo aceitáveis dois sítios, totalizando $3.000 \mathrm{ml}$ em 24 horas, quando necessário ${ }^{(10,13)}$. Cabe ao enfermeiro avaliar e observar, periodicamente, o local da punção, a fim de evitar complicações decorrentes do excesso de volume de líquido administrado por essa via.

O tempo de permanência, independentemente do cateter utilizado, pode ser de até sete dias, desde que não haja sinais flogísticos ${ }^{(12)}$. Outro autor afirma que quando não houver sinal de alteração no sítio de punção, a frequência de troca da punção dependerá da droga infundida. Drogas irritantes, como corticoides, requerem rodízio mais frequente dos sítios, enquanto, que a morfina permite a manutenção do mesmo sítio de punção por até duas semanas ${ }^{(1)}$. Deve ser obedecida a recomendação de que o novo local deve estar a uma distância mínima de cinco centímetros do local anterior $^{(1-2)}$. Contudo, os eventos adversos relacionados à hipodermóclise são raros, reversíveis e de pequena importância clínica, contanto que sejam diagnosticados precocemente $^{(17)}$. Geralmente, um edema discreto é reversível reduzindo-se velocidade de infusão. Relacionando com uma via de acesso central, as reações da hipodermóclise são mais simples e menos prejudiciais; o cateter totalmente implantado, por exemplo, além das reações supracitadas pode ocasionar deiscência da ferida operatória, migração e desconexão do cateter e necrose cutânea por pressão ${ }^{(18)}$. 


\section{CONSIDERAÇÕES FINAIS}

A pesquisa, corroborando com estudos, demonstrou que a hipodermóclise é uma técnica segura, confiável, de fácil manipulação e com o mínimo de desconforto para o paciente, porém, ainda é pouco utilizada pela equipe da instituição do estudo.

Suas limitações, como a restrição a alguns medicamentos e a infusão de grandes volumes, não devem ser itens que levem a não utilização da via. A disponibilidade de um protocolo padrão deve incentivar a adoção mais generalizada desta abordagem e minimizar as complicações. Espera-se, com este trabalho, que a hipodermóclise se expanda tanto na nossa prática local, quanto aos demais hospitais que a tem como última via de acesso a ser lembrada. Maior conhecimento da técnica impulsiona sua utilização, proporcionando uma alternativa a mais aos pacientes oncológicos, principalmente aqueles sob Cuidados Paliativos.

\section{REFERÊNCIAS}

1. Mello SHS. Hidratação subcutânea em pacientes com AIDS no Instituto de Infectologia "Emilio Ribas". [dissertação]. São Paulo (SP): Instituto de Infectologia Emílio Ribas; 2006.

2. Girondi JBR, Waterkemper R. A utilização da via subcutânea como alternativa para o tratamento medicamentoso e hidratação do paciente com câncer. REME: rev. min. enferm. 2005;9(4):348-54.

3. Ministério da Saúde (BR). Instituto Nacional de Câncer. Cuidados paliativos oncológicos: controle da dor. Rio de Janeiro: INCA; 2001.

4. Ministério da Saúde (BR). Instituto Nacional de Câncer. Terapia subcutânea no câncer avançado. Rio de Janeiro: INCA; 2009.

5. Yap LKP, Tan SH, Koo WH. Hypodermoclysis or Subcutaneous Infusion Revisited. Singapure Med J. 2001; 42(11):526-9.

6. Sasson M, Shvasrzman P. Hypodermoclysis: An alternative infusion technique. Am Fam Phys. 2001;64:1575-8.

7. Bruera E, Legris M, Kuehn N, Miller MJ. Hypodermoclysis for the administration of fluids and narcotic analgesiscs in patients with advanced cancer. J Pain Symptom Manage 1990;5(4):218-20.
8. Rochon PA, Gill SS, Litner J, Fischbach M, Goodison AJ, Gordon M. A systematic review of the evidence for hypodermoclysis to treat dehydration in older people. J Gerontol A Biol Sci Med Sci. 1997;52(3):169-76.

9. Barton A, Fuller R, Dudley N. Using subcutaneous fluids to rehydrate older people: current practices and future challenges. QJM. 2004;97:765-8.

10. Marques C, Nunes G, Ribeira T, Santos N, Silva R, Teixeira R. Terapêutica subcutânea em cuidados paliativos. Rev Port Clin Geral. 2005;21:563-8.

11. Fainsinger RL, Tara ME, Miller MJ, Bruera E, Spachynski K, Kuehn N et al. The use of hypodermoclysis for rehydration in terminally ill câncer patients. J Pain Symptom Manage. 1994;9(5):298-302.

12. Imedio EL. La infusion subcutanea continua. Dispositivos y bombas de infusion portátiles. In: Imedio EL. Enfermeria en cuidados paliativos. Madri: Médica Panamericana; 1998. p.197-208.

13. Ferry M, Dardaine V, Constas T. Subcutaneous infusion or hypodermoclysis: a practical approach. J Amer Geriatr Soc 1999;47(1):93-5.

14. Flannagan P, Bruera E, editors. M.D. Anderson's Guide to Palliative Care for Nurses. Houston: M.D. Anderson Cancer Center; 2004.

15. Steiner RN, Bruera E. Methods of hydration in Palliative Care Patients. J Palliat. Care. 1998;14(21):6-13.

16. Fonzo-Christe C, Vukasovic C, Wasilewski-Rasca AF, Bonnabry P. Subcutaneous administration of drugs in the elderly: survey of practice and systematic literature review. Palliat Med. 2005;19(3):208-19.

17. Schoueri Júnior R. Considerações sobre a importância e a pouca utilização da hipodermóclise em pacientes idosos [dissertação]. São Paulo: Faculdade de Ciências Médicas, Santa Casa de São Paulo; 2003.

18. Da Silva F, De Campos R. Complicações com o uso do cateter totalmente implantável em pacientes oncológicos: revisão integrativa. Cogitare enferm. [Internet] 2009;14(1) [acesso em 14 ago 2010]. Disponível: http://ojs.c3sl.ufpr.br/ojs2/index.php/ cogitare/article/view/14369/9676. 